[8] _ _ "Canonical representation of nonlinear discrete-time systems," in System Mod. and Feedback Theory and Applications, A. Isidori and T. J. Tarn, Eds. Boston, MA: Birkhauser, pp. 153-168, 1992.

[9] _ A unifying representation for nonlinear discrete-time and sampled dynamics," J. Math. Syst. Est. Contr., vol. 5, no. 1, pp. 103-105, 1995.

[10] _ "On the conditions of passivity and losslessness in discrete time," in Proc. ECC, Bruxelles, Belgium, 1997.

[11] _ _ "Geometric properties of a class of nonlinear discrete-time dynamics," in Proc. ECC, Bruxelles, Belgium, 1997.

\section{A Comparison Between Hankel Norms and Induced System Norms}

\author{
Wayne W. Lu and Gary J. Balas
}

\begin{abstract}
A general definition is formulated for the Hankel norms as the induced norms of a strictly proper stable linear time-invariant (LTIV) system mapping vector-valued $\mathcal{L}^{p}$-past inputs to vector-valued $\mathcal{L}^{q}$-future outputs. Some Hankel norms are derived by the maximum principle and duality. A property termed the system integral invariance is introduced by the derivation of those Hankel norms. Furthermore, the norm-induced initial conditions and the comparison between the Hankel norms and the induced system norms are also presented.
\end{abstract}

Index Terms-Hankel norm, LTIV system, norm-induced initial condition, system integral invariance.

\section{INTRODUCTION}

The Hankel norm is defined as the induced norm of a strictly proper causal (or anticausal) linear time-invariant (LTIV) system mapping vector-valued $\mathcal{L}^{2}$-past inputs to vector-valued $\mathcal{L}^{2}$-future outputs. Reference [8] extended this concept to the map from vector-valued $\mathcal{L}^{1}$-past inputs to vector-valued $\mathcal{L}^{2}$-future outputs and from vectorvalued $\mathcal{L}^{2}$-past inputs to vector-valued $\mathcal{L}^{\infty}$-future outputs. This paper gives a general definition of the Hankel norms as the induced norms of a strictly proper stable LTIV system mapping vector-valued $\mathcal{L}^{p}$ past inputs to vector-valued $\mathcal{L}^{q}$-future outputs. Some Hankel norms are presented for the usual cases of $p, q$ being $1,2, \infty$.

The Hankel operator can be interpreted as the mapping from the past input to the future output via the state $x_{o}$ at time $t=0$. The states termed the norm-induced states, or the norm-induced initial conditions, are also presented in this paper. Some implicit functions for solving the norm-induced initial conditions exhibit a property termed the system integral invariance in this paper.

A comparison between the Hankel norms and the induced system norms is also presented in this paper. First it presents a general relationship between the Hankel norm and the induced system norm. Then it is shown that both norms are identical in some cases in terms of the system property of being linear time-invariant. A

Manuscript received October 26, 1994; revised June 15, 1995 and March 25,1996 . This work was supported in part by the Graduate School of the University of Minnesota, the National Science Foundation under Grant ECS9110254, and the NASA Langley under Grant Nasa/Nag-1-1254.

W. W. $\mathrm{Lu}$ is with the Center of Control Science and Dynamical Systems, University of Minnesota, Minneapolis, MN 55455 USA (e-mail: wzlu@ee.umn.edu).

G. J. Balas is with the Center of Control Science and Dynamical Systems, University of Minnesota, Minneapolis, MN 55455 USA

Publisher Item Identifier S 0018-9286(98)06612-4. counterexample is given to show the nonexistence of the induced system norm of the map from $\mathcal{L}^{2}$ input to $\mathcal{L}^{1}$ output.

It is well known that the Hankel norm mapping $\mathcal{L}^{2}$-past inputs to $\mathcal{L}^{2}$-future outputs plays an important role in system model reduction. Other applications of the Hankel norms include estimation of the effect of the past inputs on the future outputs in an operator-theoretic way; see, e.g., [6], where a so-called "mixed induced norm" is defined, which is the Hankel norm from $\mathcal{L}^{\infty}$-past inputs to $\mathcal{L}^{1}$-future outputs.

\section{PRELIMINARIES}

Let $\mathcal{R}$ denote the field of real numbers; $\mathcal{R}^{+}$denotes the set $\{t \in \mathcal{R}: t \geq 0\}$; and $\mathcal{R}^{n}$ denotes the set of $n \times 1$ vectors with elements in $\mathcal{R}$.

For any $u: \mathcal{R} \rightarrow \mathcal{R}^{n}$ and $\tau \in \mathcal{R}^{+}, \mathcal{P}_{\tau} u$ denotes the element-byelement projection operator

$$
\left(\mathcal{P}_{\tau} u\right)(t):= \begin{cases}u(t), & t \leq \tau \\ 0, & t>\tau .\end{cases}
$$

Define $\operatorname{sgn}(u): \mathcal{R}^{n} \rightarrow \mathcal{R}^{n}$ as the element-by-element sign operator

$$
\operatorname{sgn}(u)_{i}:= \begin{cases}1, & u_{i}>0 \\ 0, & u_{i}=0 \\ -1, & u_{i}<0 .\end{cases}
$$

Let $\mathcal{L}^{p}, p \in[1, \infty]$ denote the spaces of measurable functions, $u: \mathcal{R} \rightarrow \mathcal{R}$, such that

$$
\begin{aligned}
\|u\|_{\mathcal{L} p} & :=\left(\int_{-\infty}^{\infty}|u(t)|^{p} d t\right)^{1 / p}<\infty, \quad 1 \leq p<\infty \\
& :=\underset{t \in \mathcal{R}}{\operatorname{ess} \sup }|u(t)|<\infty, \quad p=\infty
\end{aligned}
$$

Let $\ell^{r}, r \in[1, \infty]$ denote the spaces of vectors in $\mathcal{R}^{n}$ such that

$$
\begin{aligned}
\|u\|_{\ell^{r}} & :=\left(\sum_{i=1}^{n}\left|u_{i}\right|^{r}\right)^{1 / r}, \quad 1 \leq r<\infty \\
& :=\max _{1 \leq i \leq n}\left|u_{i}\right|, \quad r=\infty .
\end{aligned}
$$

Furthermore, let $\mathcal{L}^{p, r}, p, r \in[1, \infty]$ denote the spaces of vectorvalued measurable functions, $u: \mathcal{R} \rightarrow \mathcal{R}^{n}$, such that

$$
\begin{aligned}
\|u\|_{\mathcal{L}^{p, r}} & :=\left(\int_{-\infty}^{\infty}\|u(t)\|_{\ell^{r}}^{p} d t\right)^{1 / p}<\infty, \quad 1 \leq p<\infty \\
& :=\operatorname{esssup}_{t \in \mathcal{R}}\|u(t)\|_{\ell^{r}}<\infty, \quad p=\infty .
\end{aligned}
$$

Similarly, $\mathcal{L}^{p, r}(-\infty, 0)$ denotes the spaces of vector-valued measurable functions $u$ of $t$ over half of the real line $(-\infty, 0) \cdot \mathcal{L}^{p, r}(0, \infty)$ is defined in the same manner.

Let $\left(\mathcal{L}^{p, r}\right)^{*}$ denote the dual of $\mathcal{L}^{p, r}$. Then $\left(\mathcal{L}^{p, r}\right)^{*}$ can be identified with $\mathcal{L}^{q, s}$ where

$$
\frac{1}{p}+\frac{1}{q}=1 \quad \text { and } \quad \frac{1}{r}+\frac{1}{s}=1 .
$$

For $f \in \mathcal{L}^{p, r}$, and $g \in\left(\mathcal{L}^{p, r}\right)^{*}$, we define

$$
\langle f, g\rangle:=\int_{-\infty}^{\infty} f^{T}(t) g(t) d t .
$$

Let $\mathcal{S}$ be the set of the strictly proper causal stable LTIV operators with the state-space representation $\{A, B, C, 0\}$, where the row vectors of $C$ are denoted by $C=\left[\begin{array}{lll}c_{1}^{T} & \cdots & c_{p}^{T}\end{array}\right]^{T}$. Given $G \in \mathcal{S}$, 
let $G(t)$ denote the associated impulse response. Then $G$ maps $u$ to $y$ as defined by the convolution

$$
y(t)=(G u)(t)=\int_{-\infty}^{\infty} G(t-\tau) u(\tau) d \tau
$$

The causality means that $G(t)=0$ for $t<0$. It implies $\left\|g_{i j}\right\|_{\mathcal{L}^{p, r}}=$ $\left\|g_{i j}\right\|_{\mathcal{L}^{p, r}(0, \infty)}$, where $G(t)=\left\{g_{i j}(t)\right\}, 1 \leq i \leq p, 1 \leq j \leq m$. In the frequency domain

$$
\hat{y}(j w)=\hat{G}(j w) \hat{u}(j w) .
$$

Given $G \in \mathcal{S}$, the induced system norm is defined as

$$
\begin{aligned}
\|G\|_{i, q, r}^{q, s} & :=\sup _{\substack{u \in \mathcal{L}^{p, r} \\
u \neq 0}} \frac{\|G u\|_{\mathcal{L}^{q, s}}}{\|u\|_{\mathcal{L}^{p, r}}} \\
& =\sup _{\|u\|_{\mathcal{L}^{p, r}} \leq 1}\|G u\|_{\mathcal{L} q, s}<\infty
\end{aligned}
$$

where $p, q, r, s \in[1, \infty]$.

Define [8]

$$
\begin{aligned}
R & :=\int_{-\infty}^{+\infty} G^{T}(t) G(t) d t \\
& =B^{T} Q B \\
S & :=\int_{-\infty}^{+\infty} G(t) G(t)^{T} d t \\
& =C P C^{T}
\end{aligned}
$$

where $P$ and $Q$ are the controllability and observability gramians, respectively. Let $\lambda_{m}(R)$ and $d_{m}(R)$ denote the maximum eigenvalue and maximum diagonal entry of $R$, respectively.

Let $G \in \mathcal{S}$. The well-known Hankel norm is defined as

$$
\begin{aligned}
\|G\|_{H} & :=\sup _{\substack{u \in \mathcal{L}^{2}(-\infty, 0) \\
u \neq 0}} \frac{\|y\|_{\mathcal{L}^{2}(0, \infty)}}{\|u\|_{\mathcal{L}^{2}(-\infty, 0)}} \\
& =\lambda_{m}^{1 / 2}(P Q)
\end{aligned}
$$

while the $\mathcal{H}^{\infty}$ norm is

$$
\|G\|_{\mathcal{H} \infty}=\sup _{w \in \mathcal{R}} \bar{\sigma}[\hat{G}(j w)]
$$

where $\bar{\sigma}$ denotes the maximum singular value.

\section{HANKel NoRmS AND InduCED System NoRmS}

Definition 1: Suppose $G \in \mathcal{S}$ and $p, q, r, s \in[1, \infty]$. Then the Hankel norm is defined as

$$
\|G\|_{h_{p, r}^{q, s}}:=\sup _{\substack{u \in \mathcal{L}^{p, r}(-\infty, 0) \\ u \neq 0}} \frac{\|G u\|_{\mathcal{L}, s(0, \infty)}}{\|u\|_{\mathcal{L}^{p, r}(-\infty, 0)}} .
$$

In words, $\|G\|_{h, p, r}^{q, s}$ denotes the induced norm from $\mathcal{L}^{p, r}$-past inputs to $\mathcal{L}^{q, s}$-future outputs. As a special case, $p, q, r, s$ being equal to two leads to the well-known Hankel norm.

The definition (3) has the equivalent forms

$$
\begin{aligned}
\|G\|_{h_{p, r}^{q, s}} & =\sup _{\|u\|_{\mathcal{L}^{p, r}(-\infty, 0)} \leq 1}\|G u\|_{\mathcal{L} q, s(0, \infty)} \\
& =\sup _{\|u\|_{\mathcal{L}^{p, r}(-\infty, 0)}=1}\|G u\|_{\mathcal{L}^{q, s}(0, \infty)}
\end{aligned}
$$

since the Hankel operator is bounded and linear.

First, the relation between the Hankel norm and the induced system norm is given as follows.

Theorem 1: Suppose that the following induced system norm exists for $G \in \mathcal{S}$. Then

$$
\|G\|_{h, p, r}^{q, s} \leq\|G\|_{i, q, r}^{q, s}
$$

Proof: Given $u \in \mathcal{L}^{p, r}(-\infty, 0)$

$$
\begin{aligned}
\|G u\|_{\mathcal{L} q, s(0, \infty)} & \leq\|G u\|_{\mathcal{L} q, s} \\
& \leq\|G\|_{i_{p, r}^{q, s}}\|u\|_{\mathcal{L} p, r} \\
& =\|G\|_{i_{p, r}^{q, s}}\|u\|_{\mathcal{L} p, r(-\infty, 0)}
\end{aligned}
$$

In fact, an equality holds in certain cases. In the following theorem, $\|\cdot\|_{p, r}^{q, s}$ denotes both the Hankel norms and induced system norms.

Theorem 2: Given $G \in \mathcal{S}$, the Hankel norms are identical to the induced system norms for the following indicated values of $p, r, q$, and $s$ :

$$
\begin{aligned}
\|G\|_{1,2}^{2,2} & =\lambda_{m}^{1 / 2}(R) \\
\|G\|_{2,2}^{\infty, 2} & =\lambda_{m}^{1 / 2}(S) \\
\|G\|_{1,1}^{2,2} & =d_{m}^{1 / 2}(R) \\
\|G\|_{2,2}^{\infty, \infty} & =d_{m}^{1 / 2}(S) \\
\|G\|_{1,1}^{1,1} & =\max _{j} \int_{0}^{\infty} \sum_{i=1}^{p}\left|g_{i j}(t)\right| d t \\
\|G\|_{\infty, \infty}^{\infty, \infty} & =\max _{i} \int_{0}^{\infty} \sum_{j=1}^{m}\left|g_{i j}(t)\right| d t \\
\|G\|_{1,1}^{\infty, \infty} & =\max _{i, j} \sup _{t}\left|g_{i j}(t)\right| .
\end{aligned}
$$

The induced norms and equality relations in (5)-(8) were derived in [8]. The induced system norms in (9) and (10) are shown in [1]. The same techniques can be used to extend those results to the Hankel norms. The proof of (11) is quite straightforward. Hence the proofs of Theorem 2 are omitted. Note that (5) and (6), (7) and (8), (9) and (10), and (11) itself are dual in their input-output spaces.

Theorem 3: Given $G \in \mathcal{S}$

$$
\|G\|_{h_{\infty}^{1,1}}=\int_{0}^{\infty} \sum_{i=1}^{p}\left|c_{i} e^{A t} x_{o}\right| d t
$$

where

$$
\begin{aligned}
x_{o}=\int_{0}^{\infty} e^{A \tau} B \operatorname{sgn} & {\left[B^{T} e^{A^{T} \tau} \int_{0}^{\infty} e^{A^{T} t} C^{T}\right.} \\
& \left.\times \operatorname{sgn}\left(C e^{A t} x_{o}\right) d t\right] d \tau . \\
\|G\|_{h_{\infty}^{2,2}, \infty}=\left(x_{o}^{T} Q x_{o}\right)^{1 / 2} &
\end{aligned}
$$

where

$$
\begin{aligned}
x_{o} & =\int_{0}^{\infty} e^{A t} B \operatorname{sgn}\left(B^{T} e^{A^{T} t} Q x_{o}\right) d t . \\
\|G\|_{h_{2,2}^{1,1}} & =\left(x_{o}^{T} P x_{o}\right)^{1 / 2}
\end{aligned}
$$

where

$$
x_{o}=\int_{0}^{\infty} e^{A^{T} t} C^{T} \operatorname{sgn}\left(C e^{A t} P x_{o}\right) d t .
$$

Note that (12) itself, and (14) and (16) are dual in their input-output spaces. The proof of the above theorem is presented in the Appendix.

The induced system norms corresponding to $\|G\|_{h_{2}^{1, s}}$ do not exist. We may take a simple counterexample as $G(t)=e^{-t}, t \geq 0$. Let the input be $u(t)=t /(1+t)^{2}, t \geq 0$. Then $u \in \mathcal{L}^{2}$. But the output $y=-e^{-t}+1 /(1+t), t \geq 0$ is not contained in $\mathcal{L}^{1}$.

Definition 2: Suppose $G \in \mathcal{S}$ and $p, q, r, s \in[1, \infty]$. Then the norm-induced initial condition is the set as in (18), shown at the bottom of the next page. 
Theorem 4: By Definition 2, the norm-induced initial conditions corresponding to the above-discussed Hankel norms are shown as follows: $e_{k}=\left[\begin{array}{lllll}0 & \cdots & 1 & \cdots & 0\end{array}\right]^{T}$

$$
\begin{aligned}
\|G\|_{h_{1,2}^{2,2}}: & \tilde{x}_{o}=B w \\
\|G\|_{h_{2,2}^{\infty, 2}}: & \tilde{x}_{o}=P C^{T}(S)^{-1 / 2} w \\
\|G\|_{h_{1,1}^{2,2}}: & \tilde{x}_{o}=B e_{k} \\
\|G\|_{h_{2,2}^{\infty, \infty}:} & \tilde{x}_{o}=P C^{T} e_{k} / d_{m}^{1 / 2}(S) \\
\|G\|_{h}^{1,1}: & \tilde{x}_{o}=B e_{k} \\
\|G\|_{h_{\infty}^{\infty, \infty}}: & \tilde{x}_{o}=\int_{0}^{\infty} e^{A t} B \operatorname{sgn}\left(B^{T} e^{A^{T} t} C^{T} e_{k}\right) d t \\
\|G\|_{h_{1,1}^{\infty, \infty}:}: & \tilde{x}_{o}=B e_{k} \\
\|G\|_{h_{\infty}^{1,1}, \infty}: & \tilde{x}_{o}=x_{o} \text { in }(13) \\
\|G\|_{h_{\infty}^{2,2}, \infty}: & \tilde{x}_{o}=x_{o} \text { in }(15) \\
\|G\|_{h_{2,2}^{1,1}}: & \tilde{x}_{o}=P x_{o} /\left(x_{o}^{T} P x_{o}\right)^{1 / 2}, \quad x_{o} \text { in (17) } \\
\|G\|_{H}: & \tilde{x}_{o}=\sqrt{\frac{\lambda_{m}}{x_{o}^{T} Q x_{o}}} x_{o}
\end{aligned}
$$

where $w$ in (19) is the unit eigenvector corresponding to the maximum eigenvalue of $R ; w$ in (20) is the unit eigenvector corresponding to the maximum eigenvalue of $S ; k$ in (21) is any column number for which the $\mathcal{L}^{2,2}$ norm of the individual columns of $G(t)$ is maximal; $k$ in (22) is any row number for which the $\mathcal{L}^{2,2}$ norm of the individual rows of $G(t)$ is maximal; $k$ in (23) is any column number for which the $\mathcal{L}^{1,1}$ norm of the individual columns of $G(t)$ is maximal; $k$ in (24) is any row number for which the $\mathcal{L}^{1,1}$ norm of the individual rows of $G(t)$ is maximal; $k$ in (25) is any column number for which the $\mathcal{L}^{\infty, \infty}$ norm of the individual column of $G(t)$ is maximal; $x_{o}$ in (26)-(28) needs to be selected from multiple solutions of (13), (15), and (17), respectively, to achieve the Hankel norms; and $x_{o}$ in (29) is the eigenvector corresponding to the maximum eigenvalue $\lambda_{m}$ of $P Q$.

The above norm-induced initial conditions can be derived simply by applying the extremizing unit norm past inputs obtained from the derivation of the Hankel norms into Definition 2. Note that for a multivariable system, the norm-induced initial condition can be nonunique since it is possible that, for instance, two columns of $G(t)$ have the same length and both are maximal.

\section{AN EXAMPLE}

For illustrative purposes, we take a second-order plant as follows:

$$
\begin{aligned}
\dot{x}_{1} & =-x_{1}+x_{2} \\
\dot{x}_{2} & =-x_{1}-x_{2}+u \\
y & =x_{1} .
\end{aligned}
$$

The state transition matrix is

$$
e^{A t}=\left[\begin{array}{cc}
e^{-t} \cos t & e^{-t} \sin t \\
-e^{-t} \sin t & e^{-t} \cos t
\end{array}\right], \quad t \geq 0 .
$$

Since the states of the plant exhibit oscillation, we need to use numerical computation to obtain the norm-induced initial conditions shown in Theorem 3. The computational algorithm is based on the fact that if $x_{o}$ is a solution, it does not change its direction after the integral operation. First we take some points for $x_{o}$ on half of the unit circle. Note that it is easy to verify that if $x_{o}$ is a solution, so is $-x_{o}$, and that although $x_{o}$ has both direction and magnitude, only the direction plays a role in the operation of the sign function. Then we can calculate the right side of the implicit function and check the difference between the phases of the given $x_{o}$ and calculated $x_{o}$. If the phase difference goes smaller, and then larger for three consecutive pairs, there is a local extrema in the neighborhood of the second given $x_{o}$. One can continue the same procedure by refining the points of $x_{o}$ until the phase difference is smaller than a prespecified number.

The numerical computation shows that each implicit function draws an ellipsis when the given $x_{o}$ are taken on the unit circle. The norminduced initial condition splits a little off from the long axis of the ellipsis.

The computation on (13) yields $\tilde{x}_{o}=\left[\begin{array}{ll}0.512 & 0.627\end{array}\right]^{T}$. Then $\|G\|_{h 1}=0.642$. Furthermore, the other solution $x_{o}=$ $\left[\begin{array}{ll}-0.277 & 0.378\end{array}\right]^{T}$, which is a local extrema and splits a little off from the short axis of the ellipsis, results in the input-output gain 0.210 .

By (15), $\tilde{x}_{o}=\left[\begin{array}{ll}0.532 & 0.597\end{array}\right]^{T}$ gives $\|G\|_{h_{\infty}^{2}}=0.480$. The other solution $x_{o}=\left[\begin{array}{ll}-0.229 & 0.439\end{array}\right]^{T}$ leads to the gain 0.137 .

By (17), $\tilde{x}_{o}=\left[\begin{array}{ll}0.294 & 0.571\end{array}\right]^{T}$ yields $\|G\|_{h_{2}^{1}}=0.480$. The other solution $x_{o}=\left[\begin{array}{ll}-0.0266 & 0.0314\end{array}\right]^{T}$ gives the gain 0.137 .

\section{REMARKS}

The fact that the equalities hold between the Hankel norms and induced system norms in Theorem 2 can be intuitively explained as follows. For $u \in \mathcal{L}^{1, r}$, the extremizing input $\delta_{\varepsilon}(t)$, an impulse as $\varepsilon \rightarrow 0$, only takes the time $\varepsilon$ that can be arbitrarily small. For $y \in \mathcal{L}^{\infty, s}$, given any $u \in \mathcal{L}^{p, r}$, one can define time $t=0$ at the moment when $y$ achieves its peak value in terms of the fact that the system is linear time-invariant.

Each of the implicit functions in Section III is the necessary condition so that the norm-induced initial condition, if it exists, must satisfy this condition. On the other hand, the norm-induced initial condition does exist since the Hankel norms are well-defined for a stable LTIV system. Hence the norm-induced initial condition can be selected from the solutions of the implicit function to achieve the maximum gain from past inputs to future outputs.

If we define each of the implicit functions as an integral operator $I$ (nonlinear due to the sign operator) mapping $x_{o} \in \mathcal{R}^{n}$ to $x \in \mathcal{R}^{n}$, the mapping is smooth, or it has a continuous first derivative with respect to $x_{o}$. It can be easily proved by taking the derivative of $x$ in component form with respect to $x_{o_{i}}$.

For the integral operator $I, x_{o}$ defines one-dimensional subspace $V:=\left\{x \mid x=\alpha x_{o}, \forall \alpha \in \mathcal{R}\right\}$ which is invariant to the operator $I$, or $I$-invariant. More precisely, $\forall \alpha \in \mathcal{R}, I\left(\alpha x_{o}\right)=\operatorname{sgn}(\alpha) x_{o}$. The range is also contained in $V$. This invariance is termed the system integral invariance for a stable LTIV system. For the wellknown Hankel norm, the energy approach results in an eigenvector invariance to a matrix, i.e., $P Q x_{o}=\lambda x_{o}$.

The concept of the norm-induced initial condition is quite descriptive and relevant to the Hankel norm since $\tilde{x}_{o}$ plays a role in bridging past inputs to future outputs.

The well-known Hankel norm can be defined for the operator $G \in \mathcal{L}^{\infty}$ in the frequency domain. However, only the strictly causal

$$
\chi_{o}:=\left\{\tilde{x}_{o} \mid \tilde{x}_{o}=\int_{-\infty}^{0} e^{-A \tau} B u(\tau) d \tau, \text { where }\|u\|_{\mathcal{L}^{p, r}(-\infty, 0)}=1 \text { and }\|G\|_{h_{p, r}^{q, s}}=\left\|C e^{A t} \tilde{x}_{o}\right\|_{\mathcal{L}^{q, s}(0, \infty)}\right\}
$$


(or anti-causal) part of $G$ makes contribution to the Hankel norm. In the proof 3) in the Appendix, it is shown that the causal and anticausal Hankel operators equipped with any input-output norms have the identical induced norms.

We may define the Hankel norm as

$$
\|G\|_{h_{p, r}^{q, s}}:=\sup _{\tau \in \mathcal{R}+u \in \mathcal{L}^{p, r}[0, \infty)} \sup _{\tau \in G \mathcal{P}_{\tau} u \|_{\mathcal{L}^{q}, s(\tau, \infty)}} \frac{\|u\|_{\mathcal{L}^{p, r}[0, \tau)}}{\| .}
$$

In fact, two definitions (3) and (30) are equivalent in terms of the supremum in both definitions since taking $\sup _{\tau \in \mathcal{R}+}$ implies that one can search for the norm-bounded extremizing past input over half of the real line by the fact that the system is linear time-invariant. Reference [6] defined a "mixed induced norm" from $\mathcal{L}^{\infty}$-past inputs to $\mathcal{L}^{1}$-future outputs, which is a special case of (30).

\section{APPENDIX}

Proof: Proof of Theorem 3:

1) To show $\|G\|_{h_{\infty}^{1,1}, \infty}$, define the performance index as

$$
\begin{aligned}
J & :=\int_{0}^{\infty} \sum_{i=1}^{p}\left|c_{i} e^{A t} x_{o}\right| d t \\
& :=\psi\left(x_{o}\right)
\end{aligned}
$$

subject to

$$
\dot{x}=A x+B u, \quad x(-\infty)=0
$$

and

$$
\|u\|_{\mathcal{L}^{\infty, \infty}(-\infty, 0)} \leq 1
$$

We use the maximum principle to solve this problem. The maximum principle states that the necessary condition for $u$ to maximize the cost $J$ is that the Hamiltonian function must be maximized for all $t \in(-\infty, \infty)$ and for all admissible controls.

The Hamiltonian function is

$$
H=\lambda^{T}(A x+B u)
$$

where $\lambda$ is termed the costate which satisfies

$$
\begin{aligned}
\dot{\lambda} & =-H_{x}^{T}=-A^{T} \lambda, \quad t \leq 0 \\
\lambda_{o} & =\psi_{x_{o}}^{T} \\
& =\int_{0}^{\infty} e^{A^{T} t} C^{T} \operatorname{sgn}\left(C e^{A t} x_{o}\right) d t .
\end{aligned}
$$

Note that the order of the integral and partial derivative in (33) can be interchanged since the integrand is exponentially decaying for all $x_{o} \in \mathcal{R}^{n}$ and the partial derivative of the integrand with respect to $x_{o}$ is uniformly continuous for all $t \in[0, \infty)$, possibly except for the countable points due to the absolute value which can be neglected in the integral.

Since $H_{u u}=0$,

$$
u=\operatorname{sgn}\left(B^{T} \lambda\right), \quad t<0
$$

maximizes $H(x, u, \lambda)$ on the boundary. Here, $B^{T} \lambda$ is a switching function.

By convolution

$$
x_{o}=\int_{-\infty}^{0} e^{-A \tau} B u(\tau) d \tau .
$$

By simple manipulations on (31)-(36), one can get the desired results (12) and (13).
2) To show $\|G\|_{h_{\infty}^{2,2}, \infty}$, define the performance index as

$$
\begin{aligned}
J & :=x_{o}^{T} Q x_{o} \\
& :=\psi\left(x_{o}\right)
\end{aligned}
$$

subject to

$$
\dot{x}=A x+B u, \quad x(-\infty)=0
$$

and

$$
\|u\|_{\mathcal{L} \infty, \infty(-\infty, 0)} \leq 1
$$

The Hamiltonian function is

$$
H=\lambda^{T}(A x+B u) .
$$

In the same way as before, one has

$$
\begin{aligned}
\dot{\lambda} & =-H_{x}^{T}=-A^{T} \lambda, \quad t \leq 0 \\
\lambda_{o} & =\psi_{x_{o}}^{T}=2 Q x_{o} \\
u & =\operatorname{sgn}\left(B^{T} \lambda\right), \quad t \leq 0 \\
x_{o} & =\int_{-\infty}^{0} e^{-A \tau} B u(\tau) d \tau .
\end{aligned}
$$

The results of (14) and (15) follow from simple manipulations on (37)-(41).

3) We use the duality [9] to show $\|G\|_{h_{2,2}^{1,1}}$. The dual of $G$ : $\mathcal{L}^{\infty, \infty}(-\infty, 0) \rightarrow \mathcal{L}^{2,2}(0, \infty)$ is the mapping

$$
G^{*}:\left(\mathcal{L}^{2,2}(0, \infty)\right)^{*} \rightarrow\left(\mathcal{L}^{\infty, \infty}(-\infty, 0)\right)^{*} .
$$

That is

$$
G^{*}: \mathcal{L}^{2,2}(0, \infty) \rightarrow \mathcal{L}^{1,1}(-\infty, 0) .
$$

To determine the form of $G^{*}$, let $x_{o} \in \ell^{2}$ and $y \in \mathcal{L}^{2,2}(0, \infty)$, and define the mapping

$$
\begin{aligned}
\Psi_{f} & : \ell^{2} \rightarrow \mathcal{L}^{2,2}(0, \infty) \\
\left(\Psi_{f} x_{o}\right)(t) & :=C e^{A t} x_{o}, \quad t>0 .
\end{aligned}
$$

We have

$$
\left\langle y, \Psi_{f} x_{o}\right\rangle=\left\langle\Psi_{f}^{*} y, x_{o}\right\rangle
$$

where the first duality bracket is for $\mathcal{L}^{2,2}(0, \infty)$ and the second is for $\ell^{2}$. Then

$$
\begin{aligned}
\left\langle y, \Psi_{f} x_{o}\right\rangle & =\int_{0}^{\infty} y^{T}(\tau) C e^{A \tau} x_{o} d \tau \\
& =\left[\int_{0}^{\infty} e^{A^{T} \tau} C^{T} y(\tau) d \tau\right]^{T} x_{o}
\end{aligned}
$$

Hence

$$
\Psi_{f}^{*} y=\int_{0}^{\infty} e^{A^{T} \tau} C^{T} y(\tau) d \tau
$$

Let $u \in \mathcal{L}^{\infty, \infty}(-\infty, 0)$ and define the mapping

$$
\begin{aligned}
\Psi_{p} & : \mathcal{L}^{\infty, \infty}(-\infty, 0) \rightarrow \ell^{2}, \\
\Psi_{p} u & :=\int_{-\infty}^{0} e^{-A t} B u(t) d t .
\end{aligned}
$$

We have

$$
\left\langle x_{o}, \Psi_{p} u\right\rangle=\left\langle\Psi_{p}^{*} x_{o}, u\right\rangle \text {. }
$$


Then

$$
\begin{aligned}
\left\langle x_{o}, \Psi_{p} u\right\rangle & =\int_{-\infty}^{0} x_{o}^{T} e^{-A t} B u(t) d t \\
& =\int_{-\infty}^{0}\left(B^{T} e^{-A^{T} t} x_{o}\right)^{T} u(t) d t .
\end{aligned}
$$

Hence

$$
\left(\Psi_{p}^{*} x_{o}\right)(t)=B^{T} e^{-A^{T} t} x_{o}, \quad t<0 .
$$

By (42) and (43)

$$
\begin{aligned}
\left(G^{*} y\right)(t) & =\left(\Psi_{p}^{*} \Psi_{f}^{*} y\right)(t) \\
& =\int_{0}^{\infty} B^{T} e^{A^{T}(\tau-t)} C^{T} y(\tau) d \tau, \quad t<0 .
\end{aligned}
$$

Hence $G^{*}$ can be identified with the state space representation of $\left\{A^{T}, C^{T}, B^{T}, 0\right\}$.

Furthermore, the mapping $G^{*}: \mathcal{L}^{2,2}(0, \infty) \rightarrow \mathcal{L}^{1,1}(-\infty, 0)$ (anticausal) is equivalent to the mapping $G^{*}: \mathcal{L}^{2,2}(-\infty, 0) \rightarrow$ $\mathcal{L}^{1,1}(0, \infty)$ (causal) with the convolution

$$
\left(G^{*} y\right)(t)=\int_{-\infty}^{0} B^{T} e^{A^{T}(t-\tau)} C^{T} y(\tau) d \tau, \quad t>0
$$

where $y \in \mathcal{L}^{2,2}(-\infty, 0)$ since (45) can be obtained from (44) by change of variables, and vice versa. Obviously, this argument holds for the Hankel operator equipped with any other input-output norms.

Now consider the system $G^{*}$ as a mapping from $\mathcal{L}^{\infty, \infty}(-\infty, 0)$ to $\mathcal{L}^{2,2}(0, \infty)$. Its norm can be calculated by (14) and (15) while the dual system $\{A, B, C, 0\}$ mapping $\mathcal{L}^{2,2}(-\infty, 0)$ to $\mathcal{L}^{1,1}(0, \infty)$ has the same Hankel norm.

Note that the result in the proof 3 ) can also be derived by a variational approach with the definition of the performance index

$$
\begin{aligned}
J & :=\int_{0}^{\infty} \sum_{i=1}^{p}\left|c_{i} e^{A t} x_{o}\right| d t \\
& :=\psi\left(x_{o}\right)
\end{aligned}
$$

subject to

$$
\dot{x}=A x+B u, \quad x(-\infty)=0
$$

and

$$
\int_{-\infty}^{0} u^{T} u d t=1
$$

With the result obtained by this approach, a few steps of change of variables are needed to show (16) and (17). It can be shown by this approach that the implicit function is also a local sufficient condition for $x_{o}$ to achieve a local extremal input-output gain $\left(H_{u u}<0\right)$.

\section{ACKNOWLEDGMENT}

The authors would like to thank J. S. Shamma and the anonymous reviewer for their valuable comments and suggestions.

\section{REFERENCES}

[1] C. A. Desoer and M. Vidyasagar, Feedback Systems: Input-Output Properties. New York: Academic, 1975.

[2] J. Doyle, B. Francis, and A. Tannenbaum, Feedback Control Theory. New York: Macmillan, 1990.

[3] K. Glover, "All optimal Hankel-norm approximations of linear multivariable systems and their $\mathcal{L}^{\infty}$-error bounds," Int. J. Contr., vol. 39, no. 6, pp. 1115-1193, 1984

[4] B. Francis, A Course in $\mathcal{H}^{\infty}$ Control Theory. Berlin, Germany: Springer-Verlag, 1987.

[5] D. E. Kirk, Optimal Control Theory-An Introduction. Englewood Cliffs, NJ: Prentice-Hall, 1970.

[6] K. Poolla and J. S. Shamma, "Asymptotic performance through adaptive robust control," in Proc. 29th IEEE Conf. Decision Contr., 1990, pp. 3176-3181.

[7] W. Rudin, Principles of Mathematical Analysis. New York: McGrawHill, 1976.

[8] D. A. Wilson, "Convolution and Hankel operator norms for linear systems," IEEE Trans. Automat. Contr., vol. 34, pp. 94-97, Jan. 1989.

[9] K. Yoshida, Functional Analysis. New York: Springer-Verlag, 1978.

[10] K. Zhou, J. Doyle, and K. Glover, Robust and Optimal Control. Englewood Cliffs, NJ: Prentice-Hall, 1996.

[11] G. Zhu and R. Skelton, "Mixed $\mathcal{L}^{2}$ and $\mathcal{L}^{\infty}$ problems by weight selection in quadratic optimal control," Int. J. Contr., vol. 53, no. 5, pp. 1161-1176, 1991. 